\title{
Backhaul and performance optimality of joint processing schemes over the cluster area
}

\begin{abstract}
In this paper, four Joint Processing (JP) schemes have been discussed and evaluated for Dynamic Point Selection (DPS) as well as Joint Transmission (JT) over a cluster of evolved Node B (eNBs) using WINNER II channel model developed for LTE-A. The purpose of this study is developing a cooperative LTE scenario in a fixed cluster of $3 \mathrm{eNBs}$ in order to evaluate and compare the performance of these schemes for both centralized and partial cooperation. It would give us a good insight about choosing the optimum scheme which provides desired performance while putting reasonable amount of burden on the backhaul of the system. Moreover, the location dependency of JP is proved over the cluster area. As a final goal, in real-world networks, exploiting a dynamic joint processing scheme selection strategy would lead to the best performance taking care of backhaul limits. It has been proved through Simulations that, centralized joint processing has better performance for both joint transmission and dynamic point selection in comparison with the partial joint processing. Also, Dynamic point selection schemes follow the performance trend of joint transmission with less performance and lower backhaul load.
\end{abstract}

Keyword: Joint processing; Evolved Node B (eNBs); Backhaul; Joint transmissions 